\title{
Civilisations
}

Revue internationale d'anthropologie et de sciences

humaines

$48 \mid 2001$

La question de l'islam et de l'Etat à l'aube du XXIe siècle

\section{L'Islam et l'Etat}

\section{Paul Claes}

\section{(2) OpenEdition}

Journals

Édition électronique

URL : http://journals.openedition.org/civilisations/3452

DOI : 10.4000/civilisations.3452

ISSN : 2032-0442

\section{Éditeur}

Institut de sociologie de l'Université Libre de Bruxelles

\section{Édition imprimée}

Date de publication : 31 août 2001

Pagination : 7-13

ISSN : 0009-8140

\section{Référence électronique}

Paul Claes, «L'Islam et l'Etat », Civilisations [En ligne], 48 | 2001, mis en ligne le 01 août 2004, consulté

le 02 mai 2019. URL : http://journals.openedition.org/civilisations/3452 ; DOI : 10.4000/

civilisations.3452

(c) Tous droits réservés 


\section{L'ISLAM ET L'ÉTAT}

Au mois de mai 1999 se tenait à l'Université Libre de Bruxelles un colloque organisé conjointement par le Centre de Sociologie Politique et le Centre de Sociologie de l'Islam Contemporain (relevant l'un et l'autre de l'Institut de Sociologie)* avec la collaboration du Centre d'Études de l'Ethnicité et des Migrations de l'Université de Liège et l'Unité d'Anthropologie et de Sociologie de l'Université Catholique de Louvain. Le thème en était L'Islam et l'État.

Le succès rencontré auprès des spécialistes aussi bien que d'un public averti, la haute qualité des communications, l'intérêt des discussions qu'elles ont suscitées a encouragé les organisateurs à rassembler et publier les textes après remaniement par les différents auteurs à la lumière des débats du colloque. Malgré le temps mis à réaliser cette entreprise, il apparait à la lecture des pages qui suivent qu'elles n'ont rien perdu de leur pertinence et de leur actualité, prouvant par là même que la problématique couverte était fondamentale.

Le rapport de l'islam à l'État se révèle problématique, au moins sur le plan des pratiques politiques. On peut le constater dans de nombreux pays pourtant très différents, tant par leurs contextes socio-politiques que par leurs traditions historiques. Une mise en perspective comparative paraissait dès lors nécessaire pour mieux comprendre si ce caractère problématique était lié à l'islam lui-même où à d'autres facteurs.

Les organisateurs du colloque avaient donc choisi de focaliser les investigations sur trois situations idéal-typiques :

- la situation dans les États islamistes où la confusion volontariste entre le religieux et le politique tend à occulter l'autonomie relative de celui-ci.

- La situation dans les États musulmans qui se servent des «valeurs» de l'islam pour fonder leur légitimité et, par la même occasion, leur hégémonie.

- La situation dans les États laïques occidentaux partagés entre la nécessité d'intégrer leurs populations immigrées musulmanes et la crainte d'une remise en question de la séparation du religieux et du politique. 
La grande question qui sous-tendait toutes les analyses peut se formuler d'une double façon. La cohabitation entre la religion musulmane et l'État laïque démocratique (selon une vision occidentalo-centrée qui tend à se mondialiser) est-elle possible, ou l'évolution de l'islam postule-t-elle un passage obligatoire (définitif ?) par la (re)constitution d'un espace politique musulman où la religion ne serve pas seulement de prétexte et d'instrument à l'instauration d'un pouvoir autoritaire, voire oppressif ? Sans doute plusieurs voies peuvent-elles être empruntées dans la transition politique, aussi bien dans un Occident de plus en plus perméable à l'islam importé que dans un monde musulman soumis au choc de la modernité, mais faut-il que cette transition soit conçue comme un retour à des temps révolus soi-disant idylliques ou comme une adaptation de l'organisation étatique à la «nation qui change» ?

Il fallait tout d'abord s'interroger sur les relations entre l'islam et l'État au cours de l'Histoire. Dans un premier chapitre, Olivier CARRÉ s'est chargé de mettre en évidence combien était injustifiée l'assimilation de l'islam dans sa «tradition longue» à la pensée islamiste actuellement dominante. La grande tradition de la pensée politique musulmane est en effet caractérisée par le «quiétisme politique». Le courant «néo-hanbaliste» des $\mathrm{XIV}^{\mathrm{e}}$ et $\mathrm{XV}^{\mathrm{e}}$ siècles, de même que le «néo-islam» de la fin du XIX ${ }^{e}$ siècle ou l'intégrisme des années récentes récusent pour leur part la traditionnelle distinction entre le spirituel et le pouvoir. Même si l'»appareil» mondial actuel de l'islam paraît cautionner - au moins passivement - la violence externe et interne des mouvements extrémistes, de nombreux penseurs musulmans contemporains font de la fièvre islamiste actuelle un simple intermède dont la prolongation mènerait, selon l'auteur, l'islam et sa civilisation à l'autodestruction.

Robert AnciauX a ensuite étudié le seul cas historique de confrontation entre une société musulmane et un État läque : celui de la Turquie. Relevant le fait qu'il n'existe en islam aucun «pouvoir» religieux mais que le pouvoir politique y a effectivement une «tâche» religieuse à remplir, il souligne que la réaction populaire musulmane contre la laïcité vient essentiellement de ce que celle-ci s'est longtemps présentée comme anti-religieuse. Chaque phase de démocratisation du régime turc a permis une plus grande expression publique de la religiosité sans pour autant que la laïcité constitutionnelle et légale soit mise en péril. A cet égard, les intellectuels turcs musulmans ne se différencient pas fondamentalement des élites croyantes occidentales. La «synthèse turco-islamique» pourrait être une voie vers une société islamique pluraliste n'excluant pas les non-croyants de la citoyenneté.

Plusieurs cas de pays se référant explicitement à l'islamisme politique ont ensuite été évoqués. Mohammad-Reza DualiLI a tout d'abord fait le bilan de l'expérience iranienne de la «République islamique». Mélange original d'une logique théocratique (gouvernement par le clergé) et d'un dispositif démocratique (constitution, élection, etc.), le modèle iranien n'a pas réussi à s'exporter malgré l'ambition de ses dirigeants. A l'intérieur même du pays, le projet de créer le «nouvel homme islamiste» se heurte à une opposition de plus en plus ferme de la population. L'échec est patent sur les plans institutionnel, économique et sociétal, aussi bien qu'en politique internationale. L'appartenance à l'islam n'est pas fondamentalement en cause, malgré un recul très important de la pratique religieuse. Mais l'assimilation de la religion au pouvoir provoque une réaction de rejet chez les jeunes, les femmes, les artistes et beaucoup d'intellectuels. Le développement du féminisme est à cet égard remarquable, unissant dans un même effort les associations de femmes lä̈ques et islamiques. La crise existentielle du régime provoque ainsi une «sécularisation par le bas», parfois teintée de désespérance, qui reste unique dans les pays musulmans. 
Dans le cas du Pakistan, analysé par Brigitte PIQUARD, le modèle est moins pur. Cet État dont les dirigeants prétendent maintenant qu'il fut «créé pour l'Islam» — fut en réalité construit pour les musulmans par des leaders laïques. La «question islamique», posée en son temps par des mouvements opposés à l'indépendance parce que celle-ci fractionnait la communauté des croyants sur une base territoriale, a été récupérée par les gouvernements pour affermir une légitimité fort compromise. La rhétorique islamique est devenue discours d'État. Mais l'institutionnalisation et la politisation des pratiques religieuses ne parvient pas à occulter le vide de sens social et la désintégration progressive de l'État. Si la violence extrémiste apparaît à certains comme la seule méthode d'action encore disponible, d'autres développent un islam associatif appuyé sur des æuvres sociales au sein desquelles s'effectue une assimilation progressive du savoir religieux et du savoir moderne.

L'instrumentalisation de l'islam à des fins politiques et étatiques est encore plus évident en Afghanistan, comme l'a expliqué ensuite Firouzeh NAHAVANDI. Il s'agit d'ailleurs là d'une tradition séculaire dans la mesure où cette instrumentalisation fut successivement le fait des rois, soucieux de légitimer leur souveraineté sur une société multiethnique dont les références premières étaient à la fois plus restreintes (les groupes communautaires) et plus vaste (l'Umma); puis par les tribus elles-mêmes par opposition à la politique royale d'occidentalisation et de modernisme; ensuite par la résistance (guerre civile) aux Britanniques, puis aux Russes, puis au régime «athée» soutenu par ceux-ci; enfin, à des fins stratégiques évidentes, par les pays étrangers et en particulier le Pakistan, soutenu par les États-Unis, l'Arabie Saoudite et d'autres pays du Golfe. Des intérêts en tous points semblables poussent d'ailleurs à instrumentaliser l'islam de manière analogue dans les anciennes républiques soviétiques d'Asie centrale. Partout on voit l'islam, de prétexte qu'il était à l'origine, se transformer progressivement en but et devenir une idéologie politique propre à concurrencer les autres modèles du XX $\mathrm{X}^{\mathrm{e}}$ siècle, voire à jouer le rôle de balancier dans les luttes stratégiques mondiales.

Les pays du Maghreb, et en particulier le Maroc qui a servi de point de départ aux réflexions de Ahmed BENANI, ont connu une autre histoire, dont la colonisation n'a constitué qu'un aspect même s'il fut important. L'auteur souligne l'ignorance généralisée au sein des populations maghrébines, et cela bien avant la période coloniale, de la dimension intellectuelle pluraliste de la pensée islamique. Le fossé qui a de tous temps séparé la langue savante des dialectes locaux a malheureusement projeté les penseurs, écrivains et savants maghrébins dans un espace culturel extra-maghrébin, soustrayant ainsi le peuple à l'usage critique de la raison islamique. La seule raison à laquelle se sont heurtés les peuples musulmans d'Afrique du Nord fut dès lors la raison occidentale des «lumières». Inévitablement liée à la colonisation, celle-ci ne pouvait provoquer qu'un mélange d'attraction et d'aversion impropre à la création d'une citoyenneté islamique. Les souverains marocains ont établi leur légitimité à la fois sur une interprétation non critique des textes sacrés, sur un fondement contractuel à la manière de Hobbes et sur une filiation mythique avec le Prophète. S'appuyant sur un communautarisme qui transcende toujours l'individu, ils ont réussi jusqu'à présent a empêcher la mutation des «sujets» en «citoyens». Le rôle des intellectuels, aussi bien musulmans qu'occidentaux est dès lors crucial : il est indispensable de réexaminer en des termes accessibles au peuple le thème du changement social en Islam dans une perspective critique et comparative mettant en perspective réciproque d'une part les deux autres formes de pensée monothéiste (christianisme et judaïsme), d'autre part les formes de modernité illustrées bien sûr par la rationalité des «lumières» mais aussi par plusieurs courants traditionnels de la culture islamique (théologie rationnelle et libre arbitre). 
La question du rapport entre l'islam et l'État devient évidemment cruciale quand il s'agit de la création ex nihilo d'une structure étatique dans un territoire peuplé majoritairement mais pas exclusivement de musulmans : la Palestine, ou du moins ce qu'il en reste. Ici aussi, il est nécessaire de prendre du recul avant de cataloguer les différents mouvements de libération palestiniens en pacifistes (le prix Nobel !), révolutionnaires, terroristes, islamistes, pluralistes, légitimes, démocratiques ou extrémistes, selon les circonstances et les besoins. Mohammed EL B ATTIU fait un utile retour en arrière d'une cinquantaine d'années pour montrer que les «bons» et les «mauvais» représentants de la cause palestinienne - on ne dira pas du peuple palestinien puisqu'il a été longtemps de bon ton d'en nier l'existence - ont été stigmatisés ainsi pour des raisons qui n'étaient pas les leurs, et que leurs rôles se sont plusieurs fois inversés au gré de nécessites exogènes. Il montre aussi combien serait illusoire la signature d'un accord politique qui ne répondrait pas aux besoins sociaux et économiques de la population et qui mettrait les leaders palestiniens en porte-à-faux par rapport à celle-ci. La symbiose entre le pouvoir et le peuple est l'élément incontournable de la pacification en Palestine, quelles que soient les volontés d'Israël et de l'Occident.

Dans sa communication au colloque, Jean-François LEGRAIN affirmait, analyse factorielle à l'appui, que le succès de l'OLP aux élections de 1996 ne prouvait nullement que la population avait opté «rationnellement» (rational choice) pour la mise en application des accord d'Oslo et la création d'un État démocratique à l'occidentale. Le mouvement de résistance islamique, par son soutien à l'Intifada, conjugue maintenant son image de piété et ses préoccupations sociales (éducation, aide aux sinistrés, crèches, etc.) au rôle de moteur intérieur de la résistance, par rapport à un leadership politique issu de l'extérieur et plus préoccupé de problèmes territoriaux que de problèmes sociaux. En étudiant et en comparant les divers projets de loi fondamentale élaborés entre 1993 et 1997 pour l'Autorité intérimaire palestinienne, l'auteur montre l'évolution progressive des finalités attribuées à l'État: si l'OLP des années 1970 prévoyait l'édification d'un «État démocratique et non confessionnel», le texte adopté finalement par le Conseil législatif (mais jamais promulgué par Yasser Arafat) fait de l'islam «la religion officielle» de l'État en construction. Si la Palestine a été longtemps en retard d'islamisation par rapport aux États voisins, l'évolution actuelle traduit bien les changements qui s'opèrent dans la société elle-même à la suite des déceptions successives subies au cours d'un «processus de paix» prolongé à l'excès. La perspective de création d'un espace politique musulman solidaire mais aussi ouvert à la modernité, grâce à l'action d'une «diaspora» intellectuelle largement imprégnée de valeurs extra-islamiques, est-elle définitivement compromise?

Si l'on formule la question, c'est que le rapport entre islam et modernité est le plus souvent posé et pensé en termes antagoniques. Comme le souligne Jocelyne CESARI, il n'y a pourtant pas d'incompatibilité à priori. Déjà au XIX ${ }^{e}$ siècle, le choc avec un Occident techniquement puissant et porteur de nouvelles valeurs avait suscité du côté musulman une réflexion réformiste (la salafiya) visant à rationaliser les données de la foi. Cet essai d'»islamisation de la modernité», fondé sur l'ijtihad (effort d'interprétation), visait à contrecarrer les formes de tradition qui figent l'islam et le rendent inadapté aux situations de nouveauté. Réservé aux intellectuels, il se solda par un relatif échec. Mais l'entrée en force récente des musulmans dans les espaces démocratiques sécularisés crée les conditions d'une évolution en sens inverse qui aboutirait cette fois à la «modernisation de l'islam». Etre musulman en Europe, nous dit l'auteur, revient à faire sortir le lien à l'islam de son évidence, de son statut de donné communautaire, culturel ou social, pour le faire entrer dans la sphère des choix individuels et donc du questionnement. Tout en considérant la référence islamique comme un héritage 
familial et culturel inaliénable, l'immense majorité des populations immigrées la situe dorénavant dans la sphère du privé. Il s'agit là d'une véritable révolution culturelle dont les pays d'«accueil» n'ont pas vraiment conscience. Même la réaction fondamentaliste de certains musulmans d'Europe leur impose une recherche personnelle procédant d'une logique de choix individuel et de libre arbitre. L'action associative d'encadrement et de maîtrise de ces pratiques devrait éviter tout «surinvestissement» en cette démarche (surenchère, ostracisme, etc.).

Les populations immigrées gardent cependant leur image de «populations à problèmes». Elles suscitent donc de la part des responsables politiques autochtones la recherche effrénée de «médiateurs politiques privilégiés» supposés capables de résoudre à moindre frais les difficultés de cohabitation dont on ne peut (ou ne veut) pas comprendre les vraies raisons. Vincent GeISSER s'est penché sur le cas des élus (français) d'origine maghrébine qui se voient systématiquement confier par les administrations ou les partis le statut d'intermédiaires obligés dans les rapports avec les opérateurs islamiques locaux (imams, théologiens, dirigeants d'associations culturelles). Ces élites «éclairées», bien que non-pratiquantes ou pratiquantes occasionnelles, ne renient en aucune manière leur patrimoine islamique, mais en présentent une image positive idéalisée, du moins aux yeux des responsables politiques autochtones. Elles ont malheureusement moins de prestige auprès de leurs interlocuteurs musulmans qui les considèrent comme incompétentes sur les plans communautaires et religieux. On constate cependant une évolution dans le sens du rapprochement au cours de cette dernière décennie : les organisations musulmanes inscrivent de plus en plus leur action dans une démarche citoyenne permettant une islamité vécue en harmonie avec la société d' «accueil». Les élus, pour leur part, s'investissent dans les associations, s'érigeant ainsi en représentants autorisés de la communauté musulmane dans la sphère publique.

En Belgique, la question se posait autrement du fait que cet État laïque intervient de façon plus ou moins directe dans la gestion temporelle des cultes reconnus (en ce compris l'expression organisée de la libre-pensée !) : d'une part, il prend à sa charge le traitement des «ministres du culte» et l'entretien des lieux de culte; d'autre part, il définit le statut et la rémunération des professeurs de religion officiant dans son propre réseau d'enseignement; enfin il subventionne un réseau d'enseignement libre (essentiellement catholique) répondant à des critères strictement définis par lui. Il a donc paru indispensable de créer pour les musulmans de Belgique un organe représentatif officiel qui puisse servir d'interlocuteur à l'État et qui soit responsable devant lui. L'absence d'un clergé unitaire propre à l'islam obligeait à innover : le 13 décembre 1998, la population concernée était ainsi amenée à désigner par voie d'élections un «organe chef de culte» musulman, au sein duquel allait être choisi un «Exécutif des Musulmans de Belgique» qui représente officiellement depuis lors une communauté de 350.000 personnes et la deuxième religion du pays. Didier Yassine BEYENS montre bien dans sa communication les multiples conséquences politiques de ce processus original, fruit à la fois d'un long travail de recherche du consensus chez les musulmans eux-mêmes et d'un compromis avec les autorités publiques d'un «pays d'accueil» qui tend ainsi à devenir le «pays d'avenir» de cette population. Il est sans doute regrettable aux yeux des plus fervents partisans de la démocratie que la reconnaissance d'une citoyenneté politique à ces résidents de longue date (souvent plusieurs générations) n'ait pu se concevoir qu'après la reconnaissance de leur citoyenneté religieuse (au détriment d'ailleurs d'une laïcité spécifique à leur culture), mais on doit bien reconnaître que l'opération a sans nul doute contribué à l'adaptation des uns et des autres à la réalité d'une cohabitation considérée de plus en plus comme inéluctable, voire comme positive et, pourquoi pas, enrichissante. 
Mais sera-t-il possible qu'un jour ces musulmans immigrés, puis «intégrés» deviennent des Européens à part entière au point de faire partie de l' «histoire nationale des pays d'accueil»? C'est la question que pose John EADE à propos de l'Angleterre. Soulignant l'incongruité qui consiste, dans un pays dont la population «historique» est essentiellement multiethnique et multiculturelle, à décentraliser le pouvoir politique sur base ethnique et culturelle (l'Écosse, le Pays de Galles), l'auteur se demande ce que peuvent devenir les Indo-pakistanais et les noirs britanniques dans un pareil schéma (ils nous rappellent Isaac et Muhammad s'interrogeant mutuellement sur l'espace réservé aux «Belges» quand les Wallons et les Flamands auront décidé de se séparer définitivement !). La construction d'un islam «ethnicisé» correspondrait à l'exclusion racialisée de ces «étrangers» qui, s'ils conservent des allégeances à leur population d'origine et à leur communauté globale, n'en subissent pas moins la pression incessante des institutions civiles et étatiques de la société où ils se sont installés. Les jeunes musulmans britanniques participent à la construction d'une culture populaire hybride, mixée, fusionnelle, voire parodique, et rejettent le stéréotype de l'immigré traditionnel, passif et servile. Dans certaines régions du Nord, ils combinent même très aisément les composantes patriarcale et autoritaire de leur culture d'origine aux valeurs machistes des populations autochtones. La réaction des filles et des femmes musulmanes est à cet égard révélatrice, elles qui se tournent vers l'étude des textes islamiques pour mettre en cause cette fusion naissante de traditions conservatrices. Plutôt que de séparer en termes exclusivistes, le défi actuel serait de garantir au «peuple britannique» tout entier son caractère cosmopolite, diversifié, multinational, multiethnique et multiculturel. Au-delà d'une simple tolérance mutuelle, ce dont il a le plus besoin est d'un débat public sur l'intérêt commun à toutes ses composantes.

Il faudrait pour cela que l'image prégnante en Occident d'un islam incompatible avec la démocratie s'estompe au profit d'une meilleure connaissance de la diversité des courants qui s'y manifestent et de la multiplicité des acteurs qui s'en réclament. La représentation monolithique de l'islam qui prévaut chez de nombreux «démocrates» est faussée par les médias qui focalisent leur attention sur les aspects autoritaires et ultra-conservateurs de certains régimes arabes, comme sur le recours à la violence de la part de certains mouvements «intégristes» qui y voient le seul recours pour lutter contre la violence d'État. Elle est renforcée par les théories culturalistes développées dans un large segment de la littérature «scientifique» sous influence anglo-saxonne. Gema MARTIN-MuÑOz montre à quel point cette thèse ignore, voire occulte volontairement, le développement en Orient d'un islamisme réformiste s'appuyant sur une large base sociale et capable d'incarner l'alternance face à de vieilles élites gouvernantes inaptes à sortir leurs pays des crises qui les traversent. La jeune génération musulmane, qui a bénéficié de l'accès à l'éducation, tente de s'approprier les espaces et les valeurs universelles de la modernité en partant de ses propres référents islamiques qui ne sont nullement incompatibles avec la démocratisation, la participation politique, l'acceptation du pluralisme et du multipartisme. A quoi peuvent bien servir, se demande l'auteur, les versions déterministes qui font de l'islam un obstacle à la démocratie alors que ce sont les gouvernements qui s'autoproclament modernes et laïques qui entravent le développement de la démocratie dans les sociétés musulmanes ?

Dans son intervention au colloque, Tarik RAMADAN, dont la communication écrite ne nous est malheureusement jamais parvenue malgré de nombreux rappels, insistait aussi sur l'aspect caricatural d'une vision figée de l'islam. En se penchant plus spécifiquement sur la situation des musulmans d'Europe, il soulignait combien étaient rarissimes les cas où le cadre institutionnel des pays d'accueil imposerait des comportements que la conscience de ces musulmans les amènerait à refuser. Mais il insistait sur la nécessité, pour arriver à l'intégration civile et 
politique des populations immigrées, de leur reconnaitre avant tout le droit à l'intégration identitaire. Les actions de solidarité et d'éducation qui se développent au sein de ces populations doivent être comprises comme une étape indispensable à leur engagement critique vers une citoyenneté responsable et non pas comme une tentative de ruiner la démocratie de l'intérieur (à cet égard, l'orateur insistait aussi sur l'indépendance financière absolue que devaient sauvegarder les mouvements associatifs musulmans d'Europe par rapport aux gouvernements extérieurs). Mais si l'intégration par le culturel lui semblait essentielle pour l'avenir du citoyen musulman d'Europe - «pour être musulman, on n'a pas forcément un goût oriental» -, il attribuait aux élites européennes la responsabilité fondamentale de rendre ces musulmans «respectables parce que respectés». Toute référence à des conditions de réciprocité avec les pays d'origine serait dans cette optique la négation même des valeurs prônées par l'Occident.

Un colloque ne peutévidemment suffire à régler la question du rapport problématique entre l'islam et l'État. Dans les pays musulmans, le recours à l'islam à des fins politiques se généralise depuis trente ans malgré une longue tradition «quiétiste». Il va d'une instrumentalisation de la religion à des fins de légitimation des pouvoirs en place jusqu'à la justification des contestations les plus violentes de l'État. Mais il recouvre aussi les tentatives extrêmement réfléchies d'accession à la modernité pour des populations longtemps privées d'un des droits les plus fondamentaux de la culture musulmane : le libre arbitre (souvent limité, il est vrai, au libre arbitre du croyant). La référence à un code familial inégalitaire est sans doute l'aspect pratique le plus douloureux de ce retour aux sources aux multiples facettes. Dans les pays occidentaux, où l'immigration musulmane s'impose progressivement comme un fait irréversible, la réaction de l'État laïque est faite d'un mélange de crainte historico-culturelle et d'une certaine fascination. Dans leur désir de contrôler un phénomène qu'ils connaissent mal, les gouvernants européens sont tentés de «courtiser» la religion nouvelle et de la faire entrer dans l'espace public, alors même que la transition générationnelle des populations immigrées ouvre l'islam à la sécularisation. En clôture du colloque, Felice DassetTo et Marco MarTiniflLo montraient combien cette inclusion du religieux dans l'espace de l'État est susceptible de modifier la relation traditionnelle entre État et religions dans le monde européen (en matière d'application du droit, par exemple). De nombreuses réflexions sont encore nécessaires sur le sujet. Les organisateurs du colloque espèrent sincèrement avoir contribué à faire prendre conscience de leur intérêt comme de leur urgence.

Paul Claeys

Chargé de cours honoraire Ancien directeur du Centre de Sociologie politique

Note des éditeurs :

* Depuis la tenue de ce colloque, le Centre de Sociologie de l'Islam contemporain est intégré dans le Centre d'Études de la Coopération Internationale et du Développement [CECID], au sein du même Institut de Sociologie. 